\title{
Olfactory function in young adolescents with Down's syndrome
}

\author{
Donald A McKeown, Richard L Doty, Daniel P Perl, Richard E Frye, Ivy Simms, \\ Andrew Mester
}

\begin{abstract}
Decreased ability to smell is present in adults with Down's syndrome, many of whom are known to have brain pathology analogous to that seen in Alzheimer's disease. Because olfactory loss is well documented in Alzheimer's disease, the question arises whether young adolescents with Down's syndrome, who have no clear Alzheimer's disease-like neuropathology, also exhibit olfactory dysfunction. To consider this issue, standardised tests of odour discrimination and identification were administered to 20 young adolescents with Down's syndrome (mean age (SD) $13.89(1.98)$ years) and their test scores were compared with 20 mentally retarded and 20 non-mentally retarded control subjects matched to the patients with Down's syndrome on the basis of cognitive ability. No significant differences in olfactory function were found among the three study groups. These findings, along with those from studies of olfactory function in older patients with Down's syndrome, suggest that Down's syndrome related olfactory dysfunction occurs only at ages when Alzheimer's disease-like pathology is present.
\end{abstract}

(F Neurol Neurosurg Psychiatry 1996;61:412-414)

Smell and Taste Center, University of Pennsylvania, Philadelphia, PA, USA D A McKeown

R L Doty

R E Frye

I Simms

A Mester

Neuropathology Division, Mount Sinai Medical Center, New York, NY, USA D P Perl

Correspondence to: Dr Richard L Doty, Smell and Taste Center, University of Pennsylvania Medical Center, 3400 Spruce Street 5 Ravdin Pavilion, Philadelphia, PA 19104 USA.

Received 8 December 1995 and in revised form 2 May 1996 Accepted 2 May 1996
Keywords: Down's syndrome; smell; olfaction

Down's syndrome, a trisomy 21 disorder, is the most common clinical syndrome associated with mental retardation, accounting for about $17 \%$ of the retarded population. ${ }^{1}$ This syndrome is characterised by a range of physical and cognitive deficits, including a small anteroposteriorly flattened skull, a short flat bridged nose, epicanthus, short phalanges, and mild to severe mental retardation. Adults with Down's syndrome, particularly those who live beyond the age of 30 years, exhibit neuritic plaques and neurofibrillary tangles analogous to those of Alzheimer's disease. ${ }^{1-6}$ Although the appearance of significant numbers of neurofibrillary tangles is usually delayed until the third decade of life, increased deposition of amyloid in the form of senile plaques or diffuse amyloid deposits occurs in cortical brain regions associated with olfactory processing (for example, entorhinal cortex) as early as the age of 19 years. $^{7}$

Recently, several investigators have reported, on the basis of empirical testing, that adult patients with Down's syndrome have difficulty smelling, ${ }^{8-10}$ in accord with clinical findings made as early as $1928 .{ }^{11}$ However, all but one of these studies failed to compare the test scores from patients with Down's syndrome with scores from non-Down's syndrome retarded control subjects, making it possible that the deficits were due to mental retardation, rather than Down's syndrome itself. The sole study that controlled for this possibility found that 20 adult patients with Down's syndrome (mean age $=23.1$ years) scored worse on two olfactory tests (a modified version of the University of Pennsylvania smell identification test (UPSIT) ${ }^{12}$ and a yes/no odour identification test) than did 15 idiopathic mentally retarded subjects with IQs similar to those of the patients with Down's syndrome (mean age $=20.6$ years). ${ }^{9}$ In addition, the study found a weak inverse correlation between age and the modified UPSIT test scores in the Down's syndrome group ( $r=$ $-0.429, P=0.069$ ).

These findings, along with the fact that most patients with Alzheimer's disease have decreased ability to smell, led to the hypothesis that the Alzheimer's disease-like pathological changes in Down's syndrome may be the basis for the olfactory dysfunction of this disorder. If this hypothesis is correct, the olfactory function of a group of young adolescents with Down's syndrome who fall within an age range unaccompanied by meaningful Alzheimer's disease-related neuropathology should be normal. Therefore, the purpose of this study was to test the olfactory function of such adolescents with Down's syndrome and to compare their test scores with those obtained from nonDown's syndrome retarded and normal control subjects matched on the basis of cognitive ability.

\section{Methods}

SUBJECTS

Twenty of the 60 study subjects were young 
adolescents with Down's syndrome (mean age (SD) 13.89 (1.98) years; 11 boys, nine girls). Twenty others comprised a control group of retarded children matched to those with Down's syndrome on the basis of sex and mental age scores obtained on the day of testing using the Peabody picture vocabulary testrevised (PPVT-R $;^{13}$ see next section). An analogous control group of 20 non-retarded children was also formed. Twelve of these were similarly matched to the Down's syndrome group using PPVT test scores; the remainder were matched by equating their chronological age with the mental age of the matched patients with Down's syndrome. The mean (SD) chronological ages of the retarded and control groups were $13.77(4.07)$ and $5.98(1.40)$ respectively. The mean (SD) PPVT-R mental ages of the subjects tested within the three groups were $6 \cdot 11(1.98), 6.68$ $(2 \cdot 13)$, and $6 \cdot 20(1 \cdot 84)$.

The patients with Down's syndrome and the mentally retarded patients were recruited from the Westchester Association for Retarded Citizens, Westchester, New York, and the Carousel Farm Education Center, Ivyland, Pennsylvania. Twelve of the non-retarded controls were obtained from the St Cyril's of Alexandria Elementary School, East Lansdowne, Pennsylvania, and eight of the non-retarded controls were selected randomly from a database containing normal subjects maintained at the University of Pennsylvania Smell and Taste Center. Each subject's parent or legal guardian provided signed informed consent, in accord with the University of Pennsylvania's committee for the study of human beings. No remuneration was provided for participation.

\section{MATERIALS AND PROCEDURES \\ Olfactory testing}

Two well validated tests of olfactory function were given. The first test was the University of Pennsylvania smell identification test (UPSIT) $^{12}$ (commercially available as the Smell Identification Test ${ }^{\mathrm{TM}}$, Sensonics, Inc, Haddon Heights, NJ), a standardised multiple choice identification test which incorporates 40 microencapsulated odorants into an easily administered format. This test is highly reliable (test-retest $r$ from 0.90 to 0.95$)^{1415}$ and is the most common means for assessing olfactory function in North America, having been administered to over 35000 subjects over the past decade. The UPSIT has been shown to be sensitive to olfactory deficits associated with various neurodegenerative diseases, including early stage Alzheimer's disease and idiopathic Parkinson's disease, ${ }^{16-21}$ and clearly differentiates between idiopathic Parkinson's disease and progressive supranuclear palsy. ${ }^{22}$

The second test used was a 16 item three choice test of odour discrimination which does not require verbal identification of the stimuli. ${ }^{23}$ In this test, a subject is presented with 16 sets of three microencapsulated odorants (two identical, one different) on separate pages of a cardboard test booklet. The stimuli of a given page of the test are presented in rapid succes- sion and the subject is asked to select the "odd" or "different" odour from the triad. The odours of a triad were preselected to be equivalent in average perceived intensity, as determined from intensity ratings presented elsewhere. ${ }^{12}$ The number of items in which the different stimulus was reported correctly served as the dependent measure.

\section{Neuropsychological testing}

Two cognitive tests, the picture identification test (PIT) ${ }^{24}$ and the Peabody picture vocabulary test-revised (PPVT-R), ${ }^{13}$ were given. The PIT is analogous to the UPSIT except that line drawings of items representing the odorants are used instead of odorant labels. This test is designed to control for influences on UPSIT scores that may result from non-olfactory components of the test, and was used as a covariate in the UPSIT analyses of this study. The PPVT-R is a standardised, highly reliable test of vocabulary that correlates well with a wide range of standardised intelligence and vocabulary tests. In short, the child is verbally presented a word and then shown four line drawings, from which he or she must choose one that best represents the word. A mental age is derived from the raw score.

\section{Upper airway medical evaluation}

The study subjects received an anterior rhinoscopic upper airway evaluation by an experienced otorhinolaryngologist to insure that their nasal cavities were patent and to rule out diseases that might preclude valid olfactory testing. Nasal resistance was measured by anterior rhinomanometry, as described elsewhere. ${ }^{25}$

On the basis of these examinations, three non-Down's syndrome children were not included in the final study group described above. One had serious otitis in the left ear and nasal crusting within the vestibule, another had moderate bacterial rhinitis and adenoiditis, and another moderate bilateral mucosa erythema. None of the final 60 subjects evidenced health problems or nasal pathology that would have interfered with olfactory functioning.

\section{Results}

No meaningful differences among the average olfactory tests scores of the three test groups were observed (UPSIT-respective mean (SD) values for Down's syndrome, nonDown's syndrome retarded, and normal controls: $18.65(4.76) ; \quad 21.35(8.48) ; \quad 21.30$ (6.05); odour discrimination test-respective mean (SD) values: 7.40 (2.99); 7.89 (2.92); $8.08(1.93))$. An analysis of covariance (ANCOVA) was applied to the UPSIT data using PIT scores as a covariate to control for potential differences among groups in nonolfactory elements associated with the UPSIT. No effect of subject group was present $(F$ $(2,56)=0.33, P=0.74$; power coefficient $=$ $0 \cdot 80$ ), although the covariate was significant $(F(1,56)=8 \cdot 57, \mathrm{P}=0.005)$. An analogous ANCOVA applied to the odour discrimination 
scores using PPVT-R age as the covariate also found no significant group effect (group $F$ $(2,55)=0.57, P=0.57$; power coefficient $=$ $0 \cdot 72)$. The covariate was not statistically significant $(F(1,55)=2 \cdot 50, \mathrm{P}=0 \cdot 12)$.

\section{Discussion}

The present study suggests that young adolescents with Down's syndrome do not exhibit decreased olfactory function relative to control children matched on the basis of intellectual function. However, such patients exhibit-as do matched non-Down's syndrome retarded subjects-test scores of lower magnitude than non-retarded persons of their own chronological age. For example, according to published norms, ${ }^{26}$ a 14 year old non-retarded boy or girl would be expected, on average, to have an UPSIT score within the range of 34 to 38 . The respective average UPSIT scores of the patients with Down's syndrome and retarded subjects of this study fall well below this range (18.65 and 21.35 respectively). However, UPSIT scores of this magnitude fall within the low normal range for persons six years of age (the average mental age of the present subjects). Thus an UPSIT score of 19 falls at the 14th percentile of a group of 576 year olds available from our centre's UPSIT database, whereas a score of 20 falls at the 17 th percentile.

The data of the present study, along with those of the earlier Down's syndrome olfactory studies, ${ }^{8-10}$ imply that patients with Down's syndrome may first evidence major loss of olfactory function at the time when Alzheimer's diseaserelated pathology is just beginning to developthat is, in their late teens and early $20 \mathrm{~s}$. Whereas it is unlikely that neurofibrillary tangles themselves play a particular part in early Down's syndrome-related loss of olfactory function (as they typically appear in Down's syndrome 10 to 15 years after the occurrence of significant neuritic plaque formation in the early $20 \mathrm{~s}),{ }^{7}$ there is a suggestion that the olfactory function of older adults with Down's syndrome ( 32 to 54 years) may be lower than that of younger adults with Down's syndrome (20 to 31 years). ${ }^{10}$ Whether this is due to incipient dementia requires further study.

Clearly, a challenge for the future is to quantitatively establish the olfactory function of patients with Down's syndrome at all ages, to establish at what age such losses begin to appear, and to determine the pathological basis for the losses. Although the present findings, along with those from studies of olfactory function in older patients with Down's syndrome imply that Down's syndrome-related olfactory loss exists at ages when Alzheimer's disease-like pathology is present, it is conceivable that olfactory dysfunction precedes the onset of early plaque and amyloid deposition by some period of time. If so, olfactory testing may be of value in better defining and understanding such a "premorphological" period for the development of the incipient Alzheimer's disease-like brain alterations.
This work was supported by the National Institute on Deafness and Other Communication Disorders grant PO1 DC 00161 and National Institute on Aging grant RO1 AG 08148, National Institutes of Health. We thank Ms Emily Kingsley of the Westchester Association for Retarded Citizens, Westchester, NY for arranging test subjects and allowing us to test children with Down's syndrome at her estate, $\mathrm{Mr}$ and $\mathrm{Mrs}$ LaGrotte of the Carousel Farm Education Center, Ivyland, Pennsylvania, for their help in the testing of retarded children from their organisation, and Sister Agnes Patrice of St Cyril's of Alexandria Elementary School, East Lansdowne, PA, for allowing us to test volunteers within her school.

1 Mann DMA. Alzheimer's disease and Down's syndrome. Histopathology 1988;13:125-37.

2 Ball MJ. Pathological similarities between Alzheimer's disease and Down's syndrome: Is there a genetic link? Integrated Psychiatry 1987;5:159-70.

3 Fishman MA. Will the study of Down syndrome solve the riddle of Alzheimer disease? $\mathcal{F}$ Pediatr 1986;108:627-9.

4 Karlinsky H. Alzheimer's disease in Down's syndrome. A review. F Am Geriat Soc 1986;34:728-34.

5 Oliver C, Holland AJ. Down's syndrome and Alzheimer's disease-a review. Psychol Med 1986;16:307-22.

6 Price DL, Whitehouse PJ, Struble RG, Coyle JT, Clark Price DL, Whitehouse PJ, Struble RG, Coyle JT, Clark
AW, Delong MR, et al. Alzheimer's disease and Down's syndrome. Ann NY Acad Sci 1982;396:145-64.

7 Hof PR, Bouras C, Perl DP, Sparks L, Mehta N, Morrison $\mathrm{JH}$. Age-related distribution of neuropathologic changes in the cerebral cortex of patients with Down's syndrome. Arch Neurol 1995;52:379-91.

8 Warner MD, Peabody CA, Berger PA. Olfactory deficits and Down's syndrome. Biol Psychiat 1988;23:836-9.

9 Hemdal P, Corwin J, Oster H. Olfactory identification deficits in Down's syndrome and idiopathic mental retardation. Neuropsychologia 1993;31:977-84.

10 Zucco GM, Negrin NS. Olfactory deficits in Down subjects: a link with Alzheimer disease. Percept Mot Skills 1994;78:627-31.

11 Brousseau K, Brainerd HG. Mongolism: a study of the physical and mental characteristics of mongolian imbeciles. cal and mental characteristics of mon

12 Doty RL, Shaman P, Dann M. Development of the University of Pennsylvania smell identification test: a standardized microencapsulated test of olfactory function [monograph]. Physiol Behav 1984;32:489-502.

3 Dunn LM. Peabody picture vocabulary test-revised manual for forms $L$ and $M$. Circle Pines, MN: American Guidance Service, 1981

14 Doty RL, Newhouse MG, Azzalina JD. Internal consistency and short-term test-retest reliability of the University of Pennsylvania smell identification test. Chemical Senses 1985;10:297-300.

15 Doty RL, Agrawal U, Frye RE. Evaluation of the internal consistency reliability of the fractionated and whole University of Pennsylvania smell identification test (UPSIT). Perception E Psychophysics 1989;45:381-4.

16 Doty RL. Olfactory dysfunction in neurodegenerative disorders. In: Getchell TV, Doty RL, Bartoshuk LM, Snow JB Jr, eds. Smell and taste in health and disease. New York: JB Jr, eds. Smell and taste in hea

17 Doty RL, Reyes P, Gregor T. Presence of both odor identification and detection deficits in Alzheimer's disease. Brain Res Bull 1987;18:597-600.

18 Doty RL, Deems D, Stellar S. Olfactory dysfunction in Parkinson's disease: a general deficit unrelated to neurologic signs, disease stage, or disease duration. Neurology 1988;38:1237-44.

19 Doty RL, Perl DP, Steele J, Chen K-M, Pierce JD Jr, Reyes $P$, Kurland $L$. The odor identification deficit of Guam parkinsonism-dementia is equivalent to that of Alzheimer's and idiopathic Parkinson's disease. Neurology 1991;41 (suppl 2):77-80.

20 Doty RL, Stern MB, Pfeiffer C, Gollomop SM, Hurtig HI Bilateral olfactory dysfunction in early stage treated and untreated idiopathic Parkinson's disease. $\exists$ Neurol untreated idiopathic Parkinson's

21 Doty RL, Bromley SM, Stern MB. Olfactory testing as an aid in the diagnosis of Parkinson's disease: development aid in the diagnosis of Parkinson's disease: development of optimal

22 Doty RL, Golbe LI, McKeown DA, Stern MB, Lehrach $C M$, Crawford D. Olfactory testing differentiates between progressive supranuclear palsy and idiopathic Parkinson's disease. Neurology 1993;43:962-5.

23 Smith RS, Doty RL, Burlingame GK, McKeown DA. Smell and taste function in the visually impaired. Perception \& Psychophysics 1993;54:649-55.

24 Vollmecke T, Doty RL. Development of the picture identification test (PIT): a research companion to the University of Pennsylvania smell identification test. Chemical Senses 1985;10:413-4.

25 Doty RL, Deems DA, Frye R, Pelberg R, Shapiro A. Olfactory sensitivity, nasal resistance, and autonomic function in the multiple chemical sensitivities (MCS) syndrome. Arch Otolaryngol Head Neck Surg 1988;114: 1422-7.

26 Doty RL. The smell identification test administration manual, 3rd ed. Haddon Hts, NJ: Sensonics, 1995. 